\title{
Deformaciones de Segundo Orden en la Transmisión de Haces y Pulsos Gaussianos a Través de una Capa Isótropa
}

\author{
Second Order Deformations for Gaussian Beams and Pulses transmitted through \\ Isotropic Layers
}

\author{
Eduardo O. Acosta ${ }^{\# 1}$, Natalia C. Álvarez ${ }^{* 2}$, María T. Garea ${ }^{\# 3}$, Liliana I. Perez ${ }^{\# * 3}$, Patricio A. Sorichetti ${ }^{\# 5}$ \\ \# Grupo de Láser, Óptica de Materiales y Aplicaciones Electromagnéticas, Departamento de Física, Facultad de \\ Ingeniería, Universidad de Buenos Aires \\ Av. Paseo Colón 850 - (C1063ACV) CABA - Argentina \\ eacosta@fi.uba.ar \\ ${ }^{3}$ mgareadfi.uba.ar \\ ${ }^{5}$ psorichefi.uba.ar \\ * Consejo Nacional de Investigaciones Cientificas y Técnicas (CONICET)- (C1425FQB) CABA-Argentina \\ 2 nalvaredfi.uba.ar \\ ** Universidad de Buenos Aires. Consejo Nacional de Investigaciones Científicas y Técnicas. Instituto de Tecnologías y \\ Ciencias de la Ingeniería "Hilario Fernández Long". Facultad de Ingeniería. \\ Av. Paseo Colón 850 - (C1063ACV) CABA - Argentina \\ ${ }^{4}$ Iperezefi.uba.ar \\ Recibido: 13/11/17; Aceptado: 14/02/18
}

\begin{abstract}
Monochromatic plane waves allow the description of many of the properties of propagating, reflected and transmitted radiation through media of different optical properties. However, some of their characteristics only appear when considering limited beams. In this work we determine and analyse (to the second order) some characteristics of the transmission of beams (spatially limited) or pulses (limited in time), in both cases with Gaussian distribution. It is assumed that the transmission takes place through an isotropic planeparallel plate. If the beam is limited in space, we consider that the mean direction of the wave is perpendicular to the plate. On the other hand, when the beam is limited in time, we consider that all the plane waves impinge normally to the plate. We compare the non-geometric effects for both kinds of limited beams, for the cases where the plate is transparent, absorbing or with linear gain.
\end{abstract}

Keywords: Gaussian beams; Gaussian pulses; Transmission; Isotropic layers.

Resumen - Las ondas planas monocromáticas permiten describir adecuadamente muchas de las propiedades de la radiación propagada, reflejada y transmitida a través de medios de distintas propiedades ópticas,

Sin embargo, algunas características solo aparecen considerando haces y/o pulsos limitados. En este trabajo determinamos $y$ analizamos algunas características de transmisión de haces (limitados en el espacio) o pulsos (limitados en el tiempo) con distribución gaussiana. Se supone que la transmisión se realiza a través de una placa plano- paralela de material isótropo lineal y se realiza una aproximación a segundo orden. En el caso de un haz limitado en el espacio, consideramos que la dirección de onda media es perpendicular a la placa. Si está limitado en el tiempo, consideramos que la incidencia de todas las ondas es normal a la placa. Comparamos los efectos no geométricos para ambos tipos de limitación para los casos en que la placa es absorbente, transparente o con ganancia en condiciones de linealidad.

Palabras clave: Haces Gaussianos; Pulsos Gaussianos; Transmisividad.

\section{INTRODUCCIÓN}

La propagación, reflexión y transmisión de haces limitados (en el espacio o en el tiempo) con distribución gaussiana han sido muy estudiados por sus aplicaciones en la tecnología electromagnética en los rangos de microondas, submilimétrico y óptico. Como ejemplos, cabe mencionar la descripción del modo fundamental en resonadores $\mathrm{y}$ cavidades láser. Una particularidad importante es que se pueden alcanzar expresiones analíticas de las amplitudes y fases de los campos reflejado y transmitido en una aproximación a segundo orden. Esto permite un sustancial ahorro de tiempo y recursos de computación en comparación con las metodologías numéricas, particularmente en la optimización de dispositivos. Además, dada la simetría alrededor del ángulo medio de incidencia (si el haz está limitado en el espacio) o de la frecuencia 
media (si está limitado en el tiempo) es posible una interpretación sencilla de su comportamiento.

En el caso de la transmisión de haces limitados en el espacio a través de una o varias interfaces pueden evaluarse efectos no geométricos (ENGs) de primer y segundo orden longitudinales y transversales que corresponden al desplazamiento lateral (efecto Goos-Hänchen), corrimiento angular, cambio de ancho y corrimiento del punto de focalización del haz. Dependiendo de las características del haz limitado y de los medios involucrados, algunos o todos los ENGs pueden estar presentes simultáneamente. Estos efectos fueron determinados por $\mathrm{T}$. Tamir [1] y otros autores [2-6] para la reflexión de haces gaussianos limitados en el espacio en interfaces formadas por medios isótropos lineales (incluyendo multicapas), y luego fueron extendidos a la reflexión y transmisión en interfaces anisótropas [7-8] ("Método de Tamir generalizado"). En el caso de multicapas, la complejidad de los coeficientes de reflexión y transmisión dificulta (aunque no impide) la obtención de expresiones analíticas donde queden claramente determinados los cuatro ENGs a segundo orden. En cambio, las deformaciones que presentan los pulsos en la reflexión y transmisión no han sido estudiadas en profundidad aunque el desarrollo matemático es menos complejo. Según nuestro conocimiento, pese a su interés para Ensayos No Destructivos (ENDs) los estudios se limitan exclusivamente a deformaciones en la propagación a través de distintos tipos de materiales $[9,10]$.

En un trabajo anterior [11] determinamos los campos, correspondientes a haces gaussianos limitados en el espacio o en el tiempo, transmitidos a través de una capa isótropa activa inmersa en un medio isótropo transparente haciendo una aproximación a segundo orden. Si bien los medios activos son intrínsecamente no-lineales y anisótropos, la complejidad de su tratamiento lleva a usar aproximaciones de linealidad e isotropía. La primera es aceptable cuando la amplitud del campo dentro del material es pequeña (i.e. la ganancia es pequeña y no llega a la saturación). La segunda es válida cuando el campo está polarizado en uno de los modos propios. En el trabajo mencionado mostramos que la aproximación del coeficiente de transmisión es válida en muchas situaciones de interés experimental o tecnológico, teniendo en cuenta las variaciones de dicho coeficiente en el rango de trabajo.

En este trabajo aplicamos la misma metodología a haces y pulsos que se transmiten a través de capas de materiales isótropos lineales, sin limitarnos a los medios con ganancia. De esta manera mostramos que, cuando el desarrollo a segundo orden es aplicable, efectos análogos a los que aparecen para haces limitados en el espacio pueden encontrarse para pulsos limitados en el tiempo. Hacemos entonces una interpretación de cada uno de estos efectos considerando capas transparentes, absorbentes o activas inmersas en un medio transparente.

\section{HACES Y PULSOS: SUPERPOSICIÓN E INTERFERENCIA}

El haz bidimensional más simple (limitado en el espacio) consiste en la superposición de dos ondas planas con la misma frecuencia y amplitud pero distinta dirección de propagación, denominado "haz de Artmann" [12]. El resultado de esta superposición es un patrón de interferencia como la mostrada en la Fig. 1(a). Si hacemos una superposición de dos haces de Artmann tal que las normales al frente de onda estén en el mismo plano y compartan la misma normal media, obtendremos otra figura de interferencia tal que los máximos se van haciendo menos frecuentes como se muestra en las Figs. 1(b) 1(c). Como puede verse, uno de los máximos de interferencia no se desplaza a medida que se van superponiendo más haces de Artmann. Considerando que el medio es isótropo, lineal y homogéneo, los planos de interferencia constructiva son

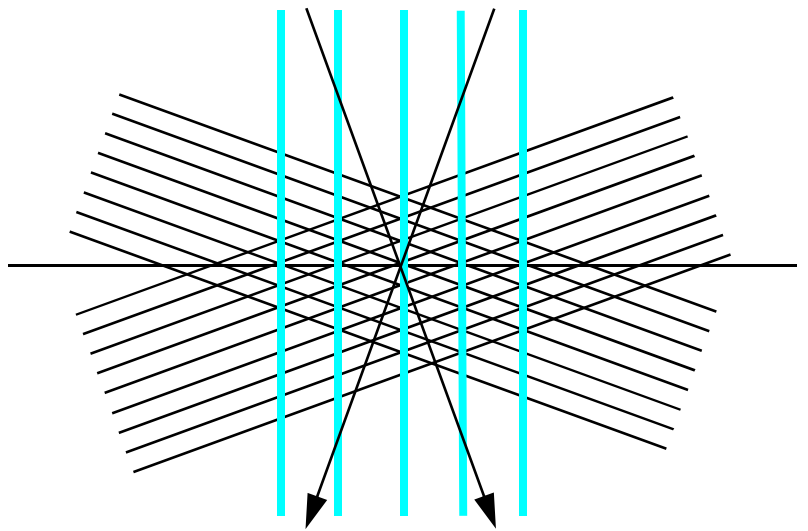

(a)

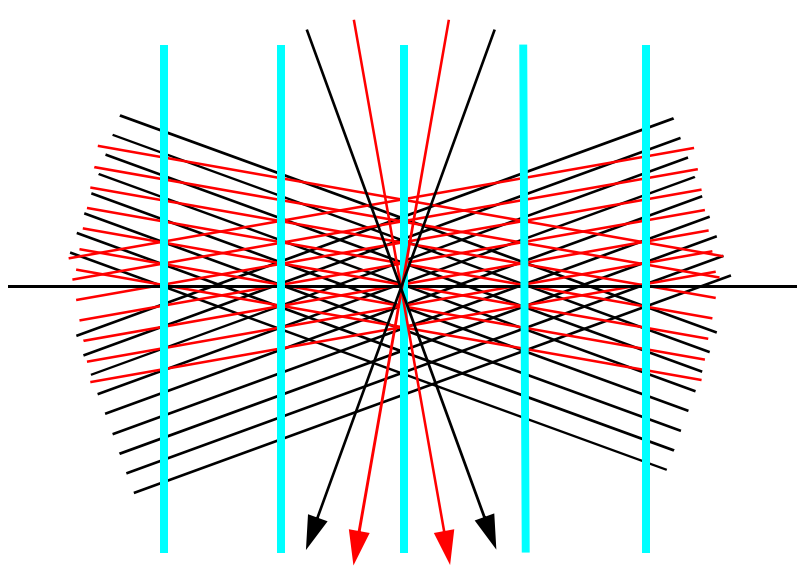

(b)

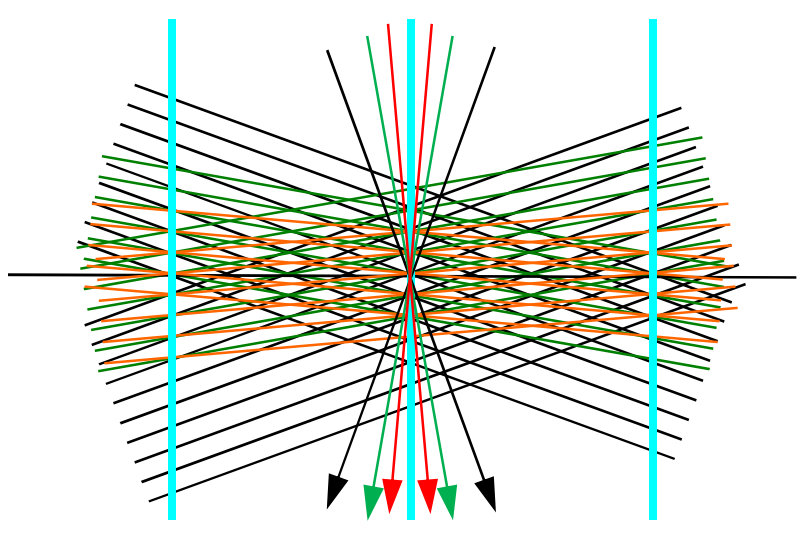

(c)

Fig. 1: Resultado de la interferencia de dos ondas planas (a) Superposición de dos b) de cuatro, c) de seis ondas monocromáticas con distintas direcciones de propagación para obtener haces limitados en el espacio. Las líneas celestes gruesas indican los planos de interferencia constructiva 
paralelos a la dirección de onda media en el primer caso (Fig. 1a). Esos máximos inalterados determinarán la dirección de propagación de la energía y el lugar de concentración de la misma. Esta es la base de la formación de haces limitados simétricos que se propagan en medios isótropos dieléctricos a través de la integral de Fourier, y ese máximo de interferencia tan especial dará la dirección del rayo asociado, es decir, la dirección de propagación de la energía del haz. Si las amplitudes de las ondas planas tienen una modulación gaussiana, el resultado será un haz gaussiano monocromático espacialmente limitado.

Análogamente, el pulso bidimensional (con simetría) más simple (limitado en el tiempo) consiste en la superposición de dos ondas planas con la misma amplitud y dirección de propagación pero distinta frecuencia. En este caso también se obtiene un patrón de interferencia. En la Fig. 2 se muestra el resultado de la superposición de tres y cinco ondas planas con la misma dirección de propagación pero distinta frecuencia de oscilación. Como se ve en la figura mencionada hay un máximo, perpendicular a la dirección de propagación, que no se altera al superponer otras ondas con otras frecuencias. Si las amplitudes de las ondas planas de distintas frecuencias tienen una distribución gaussiana, el resultado será un haz gaussiano temporalmente limitado.

\section{TRANSMISIÓN A TRAVÉS DE UNA CAPA DIELÉCTRICA}

A partir de los campos asociados a cada tipo de limitación, es posible determinar analíticamente el campo transmitido a través de una capa plano-paralela de espesor $d$ e índice de refracción $\tilde{n}$ inmersa en un medio de índice $n$ (Fig. 3). Como hemos demostrado en un trabajo anterior [11], una aproximación que ha resultado muy adecuada en muchas condiciones experimentales es la que proviene del "Método de Tamir generalizado".

Cuando el haz incidente monocromático (de frecuencia $\omega_{0}$ y longitud de onda en el vacío $\lambda_{v}$ ) posee una distribución espacial gaussiana (de semiancho $\sigma_{z}$ ), el campo eléctrico transmitido producto de la superposición de infinitas ondas planas (Fig.3) resulta

$\mathrm{E}(x, z, t)=\frac{E_{0} \sigma_{z}}{2 \sqrt{\pi}} \int_{-\infty}^{\infty} T\left(k_{x}, k_{x}^{\prime}, d\right) e^{-\frac{\sigma_{z}^{2} k_{z}^{2}}{4}} e^{i\left(k_{x}(x-d)+k_{z} z-\omega_{0} t\right)} d k_{z}$

donde $E_{0}$ es la amplitud del campo eléctrico incidente correspondiente a la onda media, las componentes de los vectores número de onda cumplen la relación $k_{x}^{2}+k_{z}^{2}=\left(2 \pi n / \lambda_{v}\right)^{2}, k_{x}^{2}+k_{z}^{\prime 2}=\left(2 \pi \tilde{n} / \lambda_{v}\right)^{2} \mathrm{y}$

$$
T\left(k_{x}, k_{x}^{\prime}, d\right)=\frac{4 k_{x} k_{x}^{\prime} e^{i k_{x}^{\prime} d}}{\left(k_{x}+k_{x}^{\prime}\right)^{2}-e^{2 i k_{x}^{\prime}}\left(k_{x}-k_{x}^{\prime}\right)^{2}}
$$

es el coeficiente de transmisión a través de la capa para cada una de las ondas. Aplicando el método de Tamir [1] para resolver analíticamente la Ec.(1) y escribiendo las expresiones en función de los índices de refracción de los medios $n$ y $\tilde{n}$, se obtiene
$\mathrm{E}(x, z, t)=E_{0} \frac{\sigma_{z}}{\sigma_{z m}} \frac{4 n \tilde{n} e^{i\left[k_{v} n(x-d)+k_{v} \tilde{n} d-\omega_{0} t\right]}}{\left[(n+\tilde{n})^{2}-e^{i 2 k_{v} \tilde{n} d}(n-\tilde{n})^{2}\right]} \mathrm{e}^{-\frac{z^{2}}{\sigma_{z m}^{2}}}$

donde $k_{v}$ representa el valor del vector número de onda en el vacío.

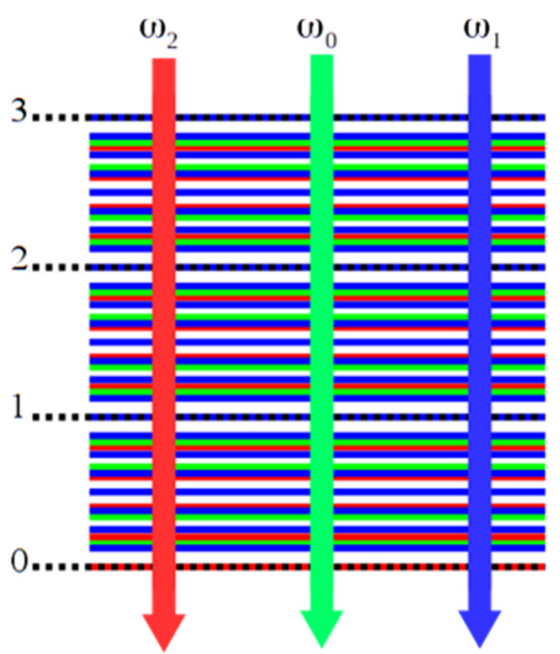

(a)

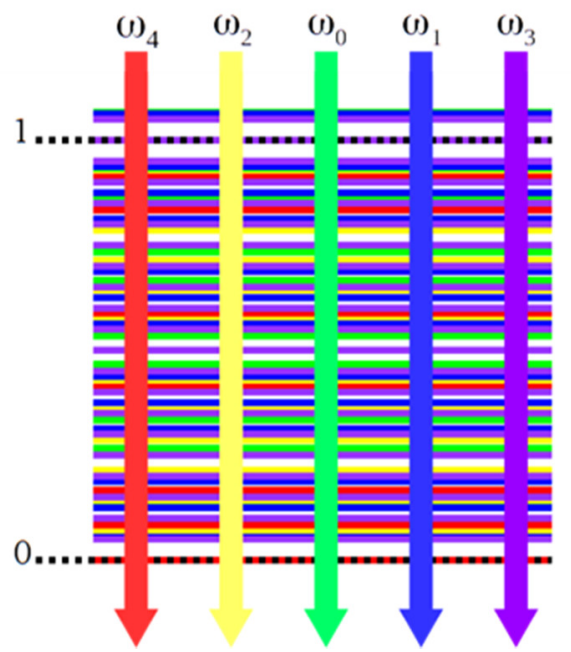

(b)

Fig. 2: Resultado de la interferencia de (a) tres y (b) cinco ondas de distintas frecuencias con la misma dirección de propagación para obtener haces limitados en el tiempo. Las líneas punteadas indican de los planos de interferencia constructiva. Los números indican el orden de interferencia constructiva.

En la Ec.(2), $\sigma_{z m}$ representa al semiancho modificado al atravesar la placa y está relacionado con el semiancho original $\sigma_{z}$ y con las características de la placa por [11]:

$$
\sigma_{z m}^{2}=\left(\sigma_{z}^{2}-i \frac{2}{k_{v} n} F\right)
$$

donde

$$
\begin{aligned}
& F=d\left[1-\frac{n}{\tilde{n}(n+\tilde{n})^{2}-e^{i 2 k_{v} \tilde{n} d}(n-\tilde{n})^{2}}\right]-x+ \\
& +i \frac{\left(n^{2}-\tilde{n}^{2}\right)^{2}\left(1-e^{i 2 k_{v} \tilde{n} d}\right)}{k_{v} n \tilde{n}^{2}\left[(n+\tilde{n})^{2}-e^{i 2 k_{v} \tilde{n} d}(n-\tilde{n})^{2}\right]}
\end{aligned}
$$


se denomina corrimiento focal. Es de hacer notar que el corrimiento focal es complejo aunque todos los medios sean dieléctricos transparentes.

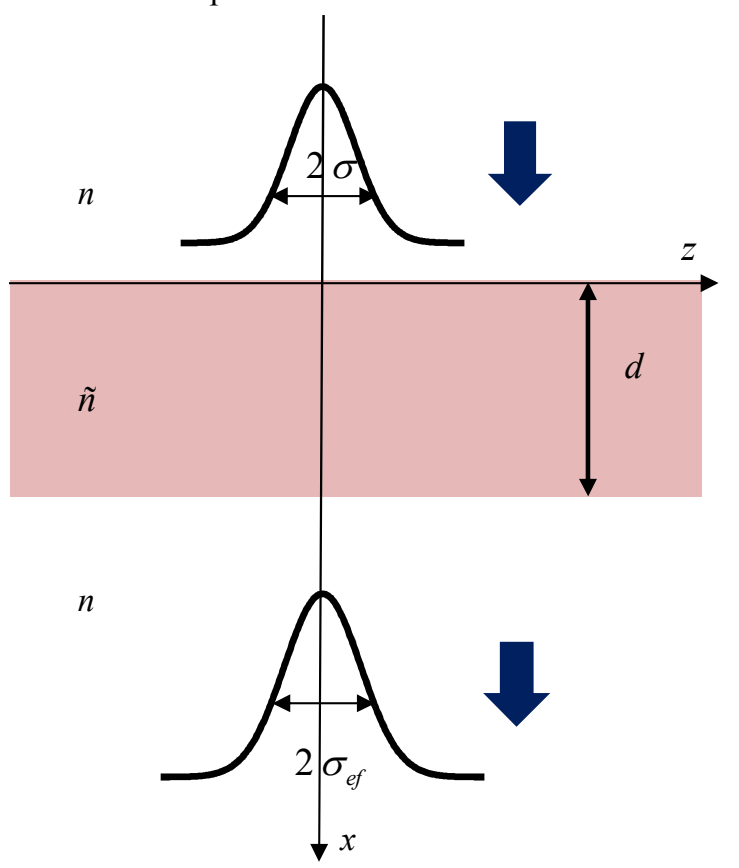

Fig. 3: Haces gaussianos incidente y transmitido a través de una lámina plano-paralela de material isótropo de índice de refracción $\tilde{n}$ inmersa en un medio de índice $n$.

De la Ec.(2) se nota claramente que el haz no sufre desplazamiento lateral ni corrimiento angular al transmitirse, independientemente de las características de los medios. Este resultado surge de la simetría del coeficiente de transmisión alrededor del ángulo medio de incidencia (incidencia normal) y de la isotropía de los medios (coincidencia entre la dirección media de propagación de la energía y la del vector número de onda).

En la Fig. 4 se grafican las amplitudes relativas de los campos transmitidos a la del campo incidente después de atravesar una placa delgada (de $100 \lambda_{v}$ de espesor) para distintos tipos de materiales. Hemos elegido la configuración de la figura para que en el caso de la capa transparente la onda media se transmita totalmente, i.e. la amplitud sea unitaria en $z=0$ y $x=d$. Esto se logra eligiendo como espesor un número entero de longitudes de onda (para incidencia normal la reflectividad resulta nula) [14]. Cuando el medio es absorbente, parte de la energía luminosa se transforma en calor, mientras que si el medio tiene ganancia, las múltiples reflexiones incrementan el campo transmitido. Como puede observarse, el máximo se encuentra en $z=0$. Es decir, no hay ENGs de primer orden.

En cambio, las diferencias en la amplitud y en la fase de las distintas ondas planas que componen el haz transmitido llevan a ENGs de segundo orden (cambios del ancho y del punto de focalización del haz).

Estos efectos provienen, fundamentalmente, de la derivada segunda del coeficiente de transmisión de la placa respecto del ángulo de incidencia. El significado del semiancho complejo $\sigma_{z m}$ queda claramente establecido cuando la parte real de $F$ es de valor despreciable frente a la imaginaria.

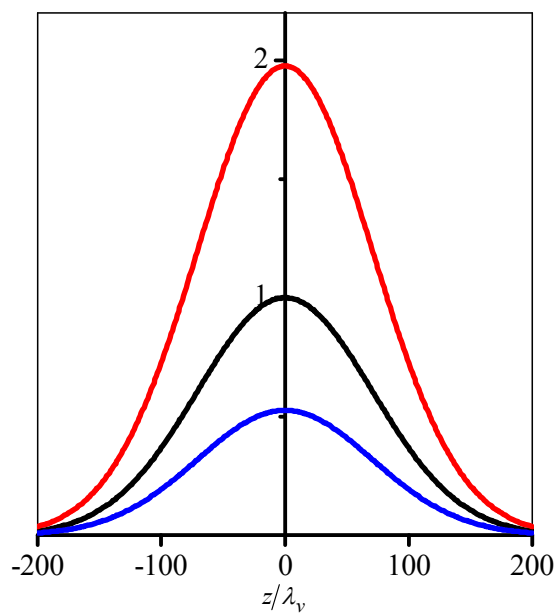

Fig. 4: Campo normalizado a la salida de la placa $(x=d)$ para $\sigma_{z}=100 \lambda_{v}$, $d=100 \lambda_{v}, n=1$ y $\tilde{n}=1,33+\mathrm{i} 0,001 \longrightarrow ; 1,33 \longrightarrow$ y $1,33-\mathrm{i} 0,001 \longrightarrow$ en función de la coordenada transversal $z / \lambda_{v}$. (en unidades de longitudes de onda)

En ese caso, la parte imaginaria de $F$ está asociada a un cambio en el ancho del haz y la real a un cambio en el punto de focalización del haz [1]. En otros casos, el ancho modificado involucra tanto a la parte real como a la imaginaria del corrimiento focal. Por otra parte, calculando el flujo del vector de Poynting de la Ec.(1) es fácil deducir que el semiancho efectivo está dado por

$$
\sigma_{z e f}=\frac{\operatorname{Re}^{2}\left(\sigma_{z m}\right)+\operatorname{Im}^{2}\left(\sigma_{z m}\right)}{\left(\operatorname{Re}\left(\sigma_{z m}^{2}\right)\right)^{1 / 2}}
$$

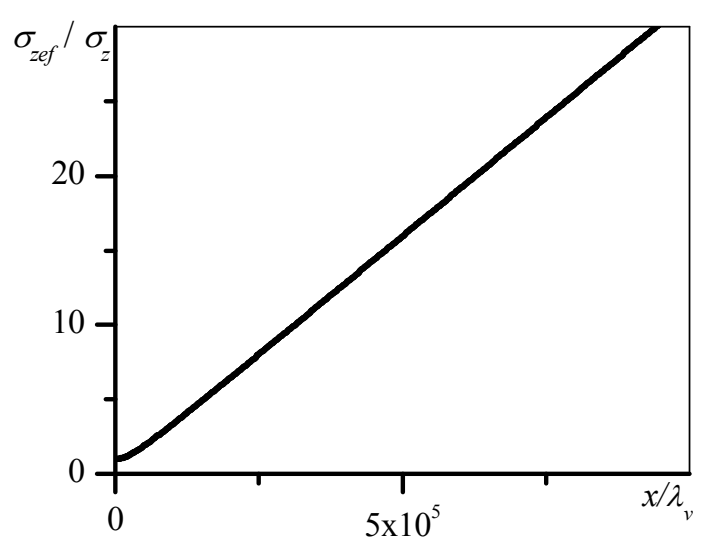

Fig. 5: Variación relativa del semiancho del haz gaussiano transmitido al propagarse en función de la distancia recorrida $\bar{x}$ para $\sigma_{\mathrm{z}}=100 \lambda_{v}, d=100 \lambda_{v}$, $n=1$ y $\tilde{n}=1,33 ; 1,33+\mathrm{i} 0,001$ y $1,33-\mathrm{i} 0,001$

En la Fig. 5 se muestra la variación $\sigma_{z e f}$ a medida que se propaga el haz después de atravesar la capa, no observándose diferencias significativas cuando la capa es transparente, activa o absorbente (dentro de los valores posibles que pueden tomar los índices de refracción). Las diferencias para los distintos tipos de medios considerados resultan menores a la milésima de longitud de onda.

En la Ref.[1] para determinar el valor del semiancho modificado $\sigma_{T}$ se considera solo la parte imaginaria del corrimiento focal $F$. Si se intentara extender este análisis para una placa como la considerada en este trabajo, el valor de $\sigma_{T}$ no diferiría de $\sigma_{z}$ a una distancia de $10000 \lambda_{v}$ de la 
placa (esta distancia corresponde aproximadamente a $5 \mathrm{~cm}$ ). Si se tiene en cuenta también la parte real del corrimiento focal el valor del semiancho $\sigma_{z}$ ef a esa distancia resulta $3,3 \sigma_{z}$ para los tres tipos de materiales. Es decir, no considerar la parte real llevaría a un error del 70\% (Fig.(6)).

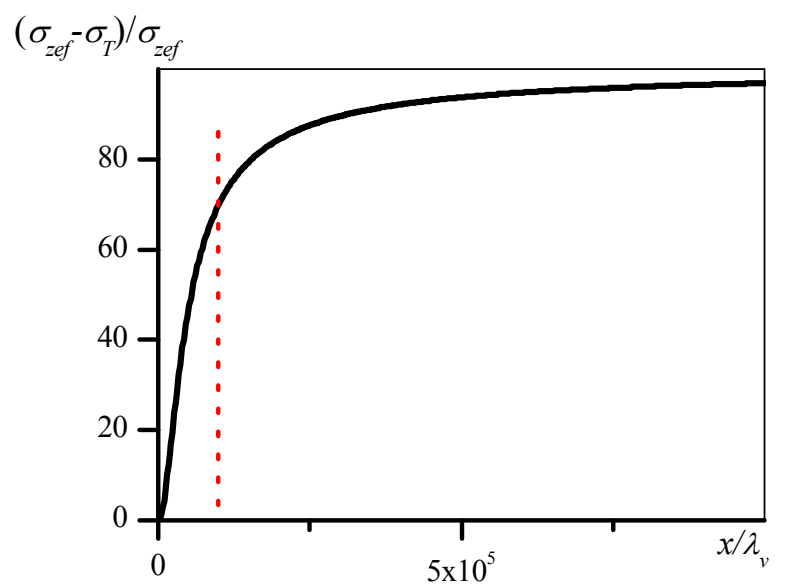

Fig. 6: Variación relativa en el cálculo del semiancho del haz gaussiano transmitido al propagarse en función de la distancia recorrida $\bar{x}$ para $\sigma_{\mathrm{z}}=100 \lambda_{v}, d=100 \lambda_{v}$, considerando solo la parte imaginaria del corrimiento focal $\left(\sigma_{T}\right)$ y su valor complejo $\left(\sigma_{z e f}\right)$. La línea punteada corresponde a $x=10000 \lambda_{v}$.

Efectos análogos a los encontrados en haces limitados en el espacio se encuentran cuando consideramos la incidencia de haces limitados en el tiempo. Considerando que el haz incidente tiene un semiancho $\sigma_{\omega}$, la frecuencia media está dada por $\omega_{0}$ y que los cambios de los índices de refracción con la frecuencia son despreciables, el campo eléctrico a la salida de la placa se puede obtener a partir de [11]

$$
\mathrm{E}(x, t)=\frac{E_{0}}{\sqrt{2 \pi} \sigma_{\omega}} \int_{-\infty}^{\infty} T(\omega, d) e^{-\frac{\left(\omega-\omega_{0}\right)^{2}}{2 \sigma_{\omega}^{2}}} e^{i \omega\left(\frac{n}{c}(x-d)-t\right)} d \omega
$$

donde $c$ es la velocidad de luz en el vacío y el coeficiente de transmisión vale

$$
T(\omega, d)=\frac{4 n \tilde{n} e^{i \omega d \frac{\tilde{n}}{c}}}{(n+\tilde{n})^{2}-e^{2 i \omega d \frac{\tilde{n}}{c}}(n-\tilde{n})^{2}}
$$

Realizando un procedimiento análogo al utilizado para haces limitados en el espacio, el campo transmitido resulta

$$
\mathrm{E}(x, t)=E_{0} T\left(\omega_{0}\right) \frac{\sigma_{\omega m}}{\sigma_{\omega}} e^{i \omega_{0}\left[\frac{n}{c}(x-d)-t\right]} \exp \left[-\frac{\sigma_{\omega m}^{2}}{2}(t-\tau)^{2}\right]
$$

donde $\tau$ representa el corrimiento temporal complejo, cuya expresión es

$$
\tau=x \frac{n}{c}+\frac{d}{c}\left(\tilde{n}^{2}-n^{2}\right) \frac{(n+\tilde{n})-(n-\tilde{n}) e^{2 i \omega_{0} d \tilde{n} / c}}{(n+\tilde{n})^{2}-(n-\tilde{n})^{2} e^{2 i \omega_{0} d \tilde{n} / c}},
$$

y el semiancho modificado complejo resulta

$$
\sigma_{\omega m}^{2}=\frac{\sigma_{\omega}^{2}}{1+\sigma_{\omega}^{2} 4 \tilde{n}^{2} \frac{d^{2}}{\left.c^{2}\left[(n+\tilde{n})^{2}-\tilde{n}^{2}\right)^{2} e^{i 2 \omega_{0} \tilde{n} d / c}(n-\tilde{n})^{2}\right]^{2}}}
$$

Se puede deducir a partir de la Ec.(7) que los efectos de primer orden están dados por la derivada primera del coeficiente de transmisión, que en este caso corresponde a un corrimiento en la frecuencia media y a un corrimiento temporal reales. Los efectos de segundo orden se traducen en un cambio en el semiancho y una modificación de la portadora reales. De la complejidad de las Ecs.(8)-(9) surge claramente que no corresponden directamente a las partes reales e imaginarias del corrimiento temporal y del semiancho complejos. Sin embargo, ambos están asociados a la asimetría del coeficiente de transmisión (en módulo y en fase) alrededor de la frecuencia media $\omega_{0}$.

En la Fig.7 se muestra el haz transmitido para tres capas plano paralelas (donde el medio es absorbente, transparente o activo) en función del tiempo considerando que $t=0$ corresponde al momento en que el haz incidente incide sobre la placa. El cálculo con $\tilde{n}=1$ representa el perfil temporal del pulso a una distancia $d$ del origen si no hubiera placa. En ella se observa que los retrasos son dependientes del tipo de material. En este ejemplo, el retraso es máximo para el medio con ganancia y mínimo para el medio con pérdidas.

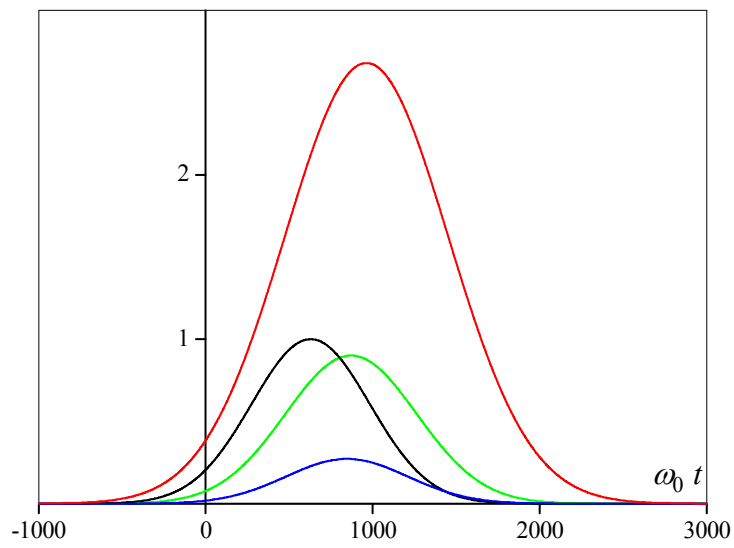

Figura 7: Campo transmitido relativo al campo incidente a la salida de la placa en función del tiempo para $n=1$ y $\tilde{n}=1-, \tilde{n}=1,33+\mathrm{i} 0,001-$; $\tilde{n}=1,33 \longrightarrow$ y $\tilde{n}=1,33-10,001 \rightleftharpoons \omega_{0} / \sigma_{\omega}=500$ y $d=200 \pi / \omega_{0}$

\section{CONCLUSIONES}

Encontramos que un haz (limitado en el espacio) que incide normalmente sobre una capa isótropa solo presenta ENGs de segundo orden, mientras que el pulso (limitado en el tiempo) presenta efectos de primer y segundo orden. Calculamos las magnitudes de dichos efectos para el caso de una placa plano-paralela de unas decenas de micrones teniendo en cuenta la existencia de múltiples reflexiones y la validez de la aproximación a segundo orden. Sin embargo, la influencia que puede tener cada uno de estos ENGs debe ser profundizada. A partir de este trabajo, consideramos que estamos en condiciones de comenzar a estudiar el caso de incidencia oblicua (donde todos los efectos estarán presentes), analizando con detalle cada uno 
de los ENGs y la influencia de considerar solo las primeras transmisiones, ya que el tamaño finito de las placas impone un número máximo. Con respecto a las deformaciones sufridas por los pulsos, el estudio deberá hacerse en forma más detallada ya que el principio de causalidad no permite que el corrimiento temporal real sea negativo.

\section{AGRADECIMIENTOS}

Este trabajo fue realizado con el apoyo parcial de los siguientes subsidios:

20020130100346BA- UBACYT 2014-2017

20020160100042BA- UBACYT 2017-2020

20020160100052BA- UBACYT 2017-2020

\section{REFERENCIAS}

[1] T. Tamir, "Nonspecular phenomena in beam fields reflected by multilayered media", J. Opt. Soc. Am. A, vol. 3, pp. 558-565, 1986.

[2] C. Chiu Chan and T. Tamir, "Beam phenomena at and near critical incidence upon a dielectric interface", J. Opt. Soc. Am. A, vol. 4, pp. 655-663, 1987.

[3] L. I. Perez, L. I. and F. Ciocci, "Nonspecular first-order effects in Kretschmann's configuration”, J. Mod. Opt., vol. 45, pp. 24872502, 1998.

[4] W. Nasalski, "Three-dimensional beam reflection at dielectric interfaces", Opt. Commun., vol. 197, pp. 217-233, 2001.

[5] A. Aiello, J. P. Woerdman, "Role of beam propagation in GoosHänchen and Imbert-Fedorov shifts", Opt. Lett., vol. 33, pp. 14371439, 2008.

[6] M. Merano, a. Aiello, M. P. van Exter, M. P. Woerdman, J. P., "Observing angular deviations in the specular reflection of a light beam", Nat. Photon., vol. 3, pp. 337-340, 2009.

[7] L. I. Perez, "Reflection and non-specular effects of 2D Gaussian beams in interfaces between isotropic and uniaxial anisotropic media", J. Mod. Opt., vol. 47, pp. 1645-1658, 2000.

[8] L. I. Perez, M. C. Simon, "Goos-Hänchen effect of an extraordinary refracted beam”, J. Mod. Opt., vol. 53, pp. 1011-1021, 2006.

[9] A. E. Siegman, Lasers, Ed. University Science Books, 1986.

[10] S. J. Orfanidis, Electromagnetic Waves and Antennas en http://www.ece.rutgers.edu/ orfanidi/ewa/, 2010

[11] V. Vázquez, J. C. Fernández, M. T. Garea, Matteo, C.L., L. I. Perez, P. A. Sorichetti, "Propagation of Gaussian beams through active layers" en Proc of SPIE, 2013, artículo $878587852 \mathrm{~W}-1$.

[12] K. Artmann, "Berechnung der Seitenversetzung des totalreflektierten Strahles”, Ann. Phys., vol. 437, pp. 87 -102, 1948.

[13] L. I. Perez, R. M. Echarri, M. T. Garea, G. D. Santiago, Determination of nongeometric effects: equivalence between Artmann's and Tamir's generalized methods", J. Opt. Soc. Am. A, vol. 28, pp. 356-362, 2011.

[14] M. Born y E. Wolf, Principles of Optic: Electromagnetic Theory of Propagation, Interference and Diffraction of Light, $7^{\mathrm{ma}}$ ed., Ed. Cambridge University Press, 1999. 